\title{
Long-term clinical outcomes up to 7- year results of intracoronary stand-alone bolus administration of eptifibatide during coronary intervention (ice) study
}

Objectives: This study evaluated the immediate, intermediate and 7-year results of intracoronary (IC) eptifibatide administration during percutaneous coronary intervention ( $\mathrm{PCl})$.

Background: Several studies tested intravenous (IV) bolus and continuous administration of eptifibatide during $\mathrm{PCl}$. However, limited data is available considering giving eptifibatide as IC bolus alone during $\mathrm{PCl}$.

Methods: Clinical outcomes of 376 patients who received coronary stent(s) + eptifibatide by three applications during PCl and were followed up for over 84 months. Group A (119 patients) had IC eptifibatide bolus only; group B (119 patients) had IC bolus and IV infusion and group C (138 patients) had IV bolus + infusion. The standard two boluses of eptifibatide $180 \mathrm{mcg} / \mathrm{kg}$ were given either via IC or IV route and only groups B and C received IV infusion at $2 \mathrm{mcg} / \mathrm{kg} / \mathrm{min}$ for $18-24$ hours.

Results: There were 256 males and 120 females, mean age $57 \pm 11$. Among them $52 \%$ were diabetics. The $6,12,24,84$-month cumulative composite endpoint of death and myocardial infraction (MI) was lower in group A (2.5\%) compared to group C (10.8\%, OR 4.3, p = 0.029), and group-B (5.8\%, OR 2.6, $p=0.17)$. Compared to group- $A$, target vessel revascularization (TVR) was three fold in group-C (OR 3.3, $p=0.001)$ and two-fold in group- $B(O R 2.0, p=0.061)$. Bleeding was significantly higher in Group-C (OR 5.4, $p<0.0001$ ) and Group-B (OR 3.4, $p=0.007$ ) compared to Group-A. Re-hospitalization was significantly lower in group A (10.9\%) compared to group B (16.8\%) and group C (28\%) ( $\mathrm{P}=0.0009)$.

Conclusion: The IC bolus alone application of eptifibatide may be safer and superior to the IV route, and continuous infusion may not be necessary. Large-scale prospective randomized trials are needed to further validate these findings.

Submitted: 19 October 2016; Accepted: 06 November 2016; Published online: 11 November 2016

Keywords: Intracoronary delivery - Eptifibatide • Intervention • Outcomes

\section{Abbreviation}

ACS: Acute Coronary Syndrome; IC: Intracoronary; PCI: Percutaneous Coronary
Intervention; IV: Intravenous; GP: Glycoprotein; MI: Myocardial Infarction; TVR: Target Vessel Revascularization; OR: Odds Ratio; CI: Confidence Interval
Walid Hassan ${ }^{*}$, Karim Fahmy Mariam Hassan', Sheref Zaghloul', Ehab I Hasan', Khaled Tammam', Atef Ibrahim1, Mohamed Thabet' ${ }^{1}$, Malik K Malik', Nathem Akhras ${ }^{2}$

'Department of Cardiovascular Disease, International Medical Center, Jeddah, Saudi Arabia

${ }^{2}$ King Faisal Heart Institute, King Faisal Specialist Hospital and Research Center, Riyadh, Saudi Arabia

*Author for correspondence:

Tel.: +966126509000

Fax: +966126509789

whassan@imc.med.sa 
Several Pathophysiological and angioscopic studies have shown that coronary thrombi play a crucial role in acute coronary syndrome (ACS) [1] and were associated with increased risk of major cardiac event during percutaneous coronary intervention (PCI) [2]. Platelet activation and aggregation play a crucial role in the pathogenesis of coronary thrombosis and deem responsible for peri-procedural ischemic complications in patients undergoing PCI [3]. Over the past decade, several clinical trials have demonstrated that the combination of an intravenous (IV) platelet glycoprotein (GP) IIb/IIIa inhibitor and low molecular weight or unfractionated heparin leads to notable reduction in periprocedural ischemic complications compared with heparin alone in patients undergoing PCI [4-10]. Although several large randomized clinical trials and meta-analysis suggest that such therapy improves longterm survival [11-13], many PCI patients worldwide do not receive a GP IIb/IIIa inhibitor, in part owing to concerns about bleeding complications and cost [14].

Small animal and clinical studies on intracoronary (IC) abciximab have been associated with intramural deposition of abciximab at the angioplasty site with effective lysis of platelet-rich thrombi [15-18]. These approaches of injecting the platelet inhibitor directly into the culprit vessel/ lesion probably achieve the maximal antiplatelet effect locally [17-19]. Intracoronary administrations of abciximab followed by 12-hour IV infusion during coronary stenting showed reduction in the incidence of major adverse cardiac events at 30 days [20], and slightly lower 6-month composite endpoint of death or myocardial infarction (MI) in IC group compared to standard IV application with similar bleeding complications [21].

One of the three GP IIb/IIIa inhibitors approved for IV use is the small molecule eptifibatide. It is a rapidly reversible, highly specific competitive inhibitor of the GP IIb/IIIa receptors. Treatment with IV eptifibatide has been shown to reduce both short-and long-term adverse cardiac events when used during PCI $[10,22,23]$. We have previously reported the immediate and intermediate results of IC standalone bolus administration of eptifibatide during coronary intervention (ICE) Study [24]. Yet, there is limited data available on the long term outcomes of IC only administration of this GP IIb/IIIa inhibitor during PCI.

So, we thought to present this study that compares the clinical outcomes of the three different modalities of eptifibatide administration that we used during PCI (IC bolus only, IC bolus + IV infusion, and IV bolus + infusion). The primary objective of the study was to compare early major adverse cardiac event (MACE), subacute stent thrombosis (SAT) and the composite endpoint of death, MI or target vessel revascularization (TVR) at 6, 12, 24 and 84 months in the three groups. The secondary objective was to analyze the incidence of immediate minor and major bleeding, and the intermediate rates of hospitalization in the three groups.

\section{Materials and Methods}

\section{Patient selection and procedure}

Three hundred seventy six consecutive patients compiled from our PCI registry from September 2001 to May 2004 who underwent coronary stent implantation and eptifibatide administration were included in the study. The research and ethics advisory committee approved the study and all patients gave written informed consent. Patients who did not have at least 84-month follow-up or who had only balloon angioplasty, rotablation or who received any IV GP IIb/IIIa inhibitors prior to the procedure were excluded from the study. All patients received standard pharmacological therapy (Table 1), including aspirin, clopidogrel (all pretreated with $300 \mathrm{mg}$ followed by 75 mg daily for one month for bare metal stents and for at least 6 months for drug eluting stents with no difference among the three strategies.), and intraprocedural unfractionated heparin according to our weigh adjusted protocol [25] to keep the activated clotting time between 200 and 250 seconds. Route of administration of eptifibatide was based on the operator's discretion according to the angiographic appearance of the target vessel (thrombus or haziness, complexity, lower TIMI flow, and bifurcation lesions). All patients received the standard two bolus doses of eptifibatide $180 \mathrm{mcg}$ $/ \mathrm{kg}$ either via IC (guiding catheter) in groups $\mathrm{A}$ and $\mathrm{B}$ (given over 60-90 seconds) or peripheral IV (group C) route, and only groups $B$ and $C$ received continuous IV infusion at $2 \mathrm{mcg} / \mathrm{kg} / \mathrm{min}$ for $18-24$ hours.

\section{Clinical parameters}

Demographic and angiographic data, coronary risk factors, medications, procedure indications, and coronary intervention data were reviewed from our PCI registry, charts and cineangiograms. The data were analyzed on immediate, intermediate and long term outcomes including minor and major bleeding, urgent revascularization, SAT, MI, death, TVR (driven by symptoms, inducible ischemia, and angiographic restenosis of $>50 \%$ of the reference diameter), and re-hospitalization. Composite endpoint of death or MI was calculated. Immediate minor and major bleeding was defined using TIMI criteria [26]. Routine follow-up hemoglobin and diligent examination to rule out significant bleeding were done in all patients. In-hospital MI was defined as the presence of new significant ST elevation or Q waves in two or more 
contiguous electrocardiographic leads or an elevation of creatine kinase and its $\mathrm{MB}$ isoenzyme to at least three times the normal reference in three samples collected at 8 hour intervals (done in all patients). Subsequent MI was defined as the development of new ST elevation or Q-waves or an elevation of creatine kinase or its MB isoenzyme to at least two times the normal reference [27]. All patients had complete clinic follow-up including stress Sesta-MIBI tests and telephone contact was made if any follow-up data were missing.

\section{Quantitative coronary angiography}

Reference diameter, minimum lumen diameter (MLD), lesion length, and residual stenosis were assessed by quantitative coronary angiography by two independent reviewers using an automated computerbased system CAAS II system (QCA for Research 1.3, Pie Medical Imaging, Maastricht, The Netherlands). Angiographic success was defined as a final angiographic residual stenosis of $<20 \%$, procedural success was considered in case of angiographic success and no inhospital major complication (acute MI, need for bypass surgery or repeat PCI, or death) occurred. Quantitative angiography was repeated whenever indicated at followup. A restenosis was defined as a MLD in the treated artery of $>50 \%$ of the reference diameter.

\section{Statistical analysis}

The chi-square or Fisher's exact test was used to compare the three groups on qualitative variables. The ANOVA was used to compare their means on quantitative variables. The Logistic regression was used to compare the three groups on outcomes adjusting for other related factors. A 5\% level of significance was used for all statistical tests. SAS version 9.1 was used for statistical analysis (SAS Institute, Cary, North Carolina, USA).

\section{Results}

\section{Patient characteristics}

The three groups were comparable in their baseline demographic characteristics and coronary artery disease risk factors prevalence. Furthermore, indications for PCI were similar for all groups, the majority being non-ST elevation MI (NSTEMI)/unstable angina (UA) acute coronary syndrome (ACS). Overall 52\% of patients were diabetic and $20 \%$ were treated with insulin. The mean ejection fraction was similar in groups $\mathrm{B}, \mathrm{C}$ and slightly lower in group $A(P=0.016)$, all other baseline characteristics were similar in the three groups except for slight older age in IC group (Table 1).

Table 1: Patient Characteristics of Treated Groups.

\begin{tabular}{|c|c|c|c|c|}
\hline GROUP & $A(N=119)$ & $B(N=119)$ & $C(\mathrm{~N}=138)$ & P VALUE \\
\hline Age, Mean \pm SD & $58.9 \pm 11.9$ & $58.1 \pm 11.2$ & $54.9 \pm 10.9$ & $0.011^{*}$ \\
\hline Male, $\%$ & $79(66.4)$ & $82(68.9)$ & $94(68.1)$ & 0.894 \\
\hline Hypertension, \% & $79(66)$ & $73(61.3)$ & $88(63.7)$ & 0.640 \\
\hline Hyperlipidemia, \% & $84(70.5)$ & $79(66.3)$ & $93(67.4)$ & 0.608 \\
\hline Smoking, \% & $50(42)$ & $44(37)$ & $47(34)$ & 0.288 \\
\hline Diabetes, $\%$ & $63(53)$ & $60(50.4)$ & $68(49.2)$ & 0.156 \\
\hline LVEF, \% & $48 \pm 12$ & $50 \pm 11$ & $50 \pm 9$ & $0.016^{*}$ \\
\hline \multicolumn{5}{|l|}{ Indication } \\
\hline Stable angina, $\%$ & $32(27)$ & $34(28.6)$ & $44(31.8)$ & \multirow{4}{*}{0.956} \\
\hline ACS/unstable angina, $\%$ & $48(40)$ & $52(43.6)$ & $54(39.1)$ & \\
\hline ACS/NSTEMI, $\%$ & $20(16.8)$ & $18(15.1)$ & $22(15.9)$ & \\
\hline STEMI, \% & $19(16)$ & $17(14.2)$ & $20(14.5)$ & \\
\hline \multicolumn{5}{|l|}{ Medications } \\
\hline ASA, $\%$ & $118(99)$ & $117(98.3)$ & $135(98)$ & 0.842 \\
\hline B Blocker, \% & $110(92)$ & $109(91.5)$ & $123(89.1)$ & 0.661 \\
\hline Statin, \% & $110(92)$ & $110(92)$ & $129(93.5)$ & 0.931 \\
\hline ACE I, \% & $88(74)$ & $92(77)$ & $108(78)$ & 0.853 \\
\hline Clopidogrel, \% & $112(94)$ & $115(96.6)$ & $131(95)$ & 0.839 \\
\hline Nitrate, $\%$ & $66(55)$ & $71(59.6)$ & $87(63)$ & 0.399 \\
\hline Insulin, \% & $25(21)$ & $18(15)$ & $22(16)$ & 0.265 \\
\hline $\mathrm{OHG}, \%$ & $50(42)$ & $41(34.4)$ & $50(36)$ & 0.388 \\
\hline
\end{tabular}

* = Statistically significant

$\mathrm{LVEF}=$ left ventricular ejection fraction

ACS $=$ acute coronary syndrome, NSTEMI = non ST elevation myocardial infarction, STEMI $=$ ST elevation myocardial infarction $\mathrm{OHG}=$ oral hypoglycemic agents 


\section{Procedure data}

The angiographic and procedural characteristics are shown in Table 2. Stent implantation was the treatment modality in all patients. The IC group had a higher percentage of patients with coronary thrombus, lower preprocedural TIMI III flow and more bifurcation lesions, but this was not statistically significant $(\mathrm{p}=$ $0.360,0.22$ and 0.48 respectively). The left anterior descending artery was most frequently intervened on, followed by the right coronary artery then left circumflex artery and vein graft. Drug-eluting stents were used in $32-40 \%$ according to stent availability during the study period (Table 2). The IC administration of eptifibatide was well tolerated by all patients in the study. There were no arrhythmias, significant bradycardia or heart block noted during IC eptifibatide administration.

\section{Clinical outcomes}

None of the study groups required urgent or elective coronary bypass surgery. There was higher TIMI III flow achieved in-IC group $(p=0.037)$. There was no acute or subacute thrombosis (SAT) in group A, while two patients in group B and three patients in group C had SAT. There was a statistically significant difference in the composite endpoint of death or MI between the IC and IV groups ( $P=0.0016$, using multiple logistic regression with stepwise selection of variables identified (age, LVEF, post PCI TIMI flow) as predictors for death or MI; Tables 1-4) in favor of IC group A. In IC group A there was no periprocedural MI, one patient had MI in the first 30 days and two patients had MI at 6 months. In-group B, two patients had periprocedural MI, two patients had MI at 30 days and two patients had MI at 6 months. None of groups A or B had MI at 12, 24 or 84 months. There was only one death at 6 months in group B. In IV group C, five patients had periprocedural MI; three had MI at 30 days, four at 6 months, and one at 12 months. There were two deaths in the IV group, at 40 days and 10 months, respectively. The majority of the MIs were NSTEMI; only one of group B and two of group C were Q-wave MI. The response of group $\mathrm{A}$ showed a significant difference from the response of each of group B and group C (p $=0.0026)$. The cumulative odds of death or MI for the

\begin{tabular}{|c|c|c|c|c|}
\hline GROUP & $A(N=119)$ & $B(\mathrm{~N}=119)$ & $C(\mathrm{~N}=138)$ & $\begin{array}{c}P \\
\text { VALUE }\end{array}$ \\
\hline Thrombus, \% & $32(26.9)$ & $24(20)$ & $36(26)$ & 0.360 \\
\hline BMS, \% & $81(68)$ & $72(60)$ & $87(63)$ & \multirow{2}{*}{0.394} \\
\hline DES, \% & $38(32)$ & $47(40)$ & $51(37)$ & \\
\hline \multicolumn{5}{|l|}{ TIMI III, \% } \\
\hline Pre & $76(63.8)$ & $79(66.4)$ & $95(68.8)$ & 0.221 \\
\hline Post & $117(98)$ & $109(91.6)$ & $125(90.5)$ & $0.037^{*}$ \\
\hline \multicolumn{5}{|l|}{ Treated vessel } \\
\hline LAD, \% & $54(45)$ & $61(51)$ & $64(46.4)$ & \multirow{6}{*}{0.848} \\
\hline LCX, \% & $24(20)$ & $23(19.3)$ & $31(22.5)$ & \\
\hline $\mathrm{RCA}, \%$ & $32(26.9)$ & $30(25.2)$ & $36(26)$ & \\
\hline SVG, \% & $6(5)$ & $4(3.4)$ & $6(4.3)$ & \\
\hline LMN, \% & $2(1.7)$ & 0 & $1(0.7)$ & \\
\hline LIMA, \% & $2(1.7)$ & 0 & $1(0.7)$ & \\
\hline \multicolumn{5}{|l|}{ Lesion type } \\
\hline $\mathrm{A}, \%$ & 18 & 20 & 21 & 0.785 \\
\hline $\mathrm{B} 1, \%$ & 33 & 31 & 32 & 0.962 \\
\hline $\mathrm{B} 2, \%$ & 50 & 47 & 48 & 0.923 \\
\hline$C, \%$ & 48 & 45 & 44 & 0.765 \\
\hline Reference diameter (mm) & $2.70 \pm 0.58$ & $2.71 \pm 0.54$ & $2.71 \pm 0.61$ & 0.82 \\
\hline Length of lesion (mm) & $16.38 \pm 10.72$ & $16.41 \pm 10.12$ & $15.98 \pm 11.08$ & 0.46 \\
\hline \multicolumn{5}{|l|}{ Minimal lumen diameter (mm) } \\
\hline Pre-procedure & $0.91 \pm 0.32$ & $0.92 \pm 0.51$ & $0.94 \pm 0.45$ & 0.34 \\
\hline Post-procedure & $2.73 \pm 0.56$ & $2.74 \pm 0.58$ & $2.76 \pm 0.61$ & 0.47 \\
\hline Bifurcation stenting, \% & $11(9)$ & $8(6.7)$ & $9(6.5)$ & 0.480 \\
\hline Number of stents + SD & $2 \pm 1.2$ & $1.9 \pm 1.3$ & $2 \pm 1.2$ & 0.544 \\
\hline Angiographic success, $\%$ & $117(98)$ & $116(97.4)$ & $134(97)$ & 0.630 \\
\hline
\end{tabular}

* = Statistically significant BMS = bare metal stent DES = drug-eluting stent $\mathrm{LAD}=$ left anterior descending, $\mathrm{LCX}=$ left circumflex, $\mathrm{RCA}=$ right coronary artery, SVG = saphenous vein graft, $\mathrm{LMN}=$ left main artery, LIMA = left internal mammary artery 


\begin{tabular}{|c|c|c|c|c|}
\hline GROUP & $A(N=119)$ & $B(\mathrm{~N}=119)$ & $C(\mathrm{~N}=138)$ & P VALUE \\
\hline Subacute Thrombosis, n (\%) & 0 & $2(1.7)$ & $3(2.2)$ & 0.292 \\
\hline \multicolumn{5}{|l|}{ Periprocedural to 6 months } \\
\hline Early MACE n (\%) & $1(0.8)$ & $4(3.3)$ & $8(5.8)$ & 0.092 \\
\hline $\mathrm{Ml}, \mathrm{n}(\%)$ & $3(2.5)$ & $6(5)$ & $12(8.7)$ & 0.095 \\
\hline TVR, n (\%) & $8(6.7)$ & $11(9.2)$ & 24(17.4) & $0.018^{*}$ \\
\hline Death, n (\%) & 0 & $1(0.8)$ & $1(0.7)$ & 0.623 \\
\hline Composite Death \& MI, n (\%) & $3(2.5)$ & $7(5.9)$ & $13(9.4)$ & 0.070 \\
\hline Rehospitalization, n (\%) & $13(10.9)$ & $20(16.8)$ & $38(28)$ & $0.0009^{*}$ \\
\hline Major Bleed, n (\%) & $3(2.5)$ & $9(7.6)$ & $13(9.4)$ & 0.163 \\
\hline Minor Bleed, n (\%) & $7(5.8)$ & $14(11.7)$ & $24(17)$ & $0.018^{*}$ \\
\hline \multicolumn{5}{|l|}{12 Months (Cumulative) } \\
\hline TVR, n (\%) & 14(11.8) & $17(14.3)$ & $42(30.4)$ & $0.0002^{*}$ \\
\hline Ml, n (\%) & $3(2.5)$ & $6(5)$ & $13(9.4)$ & 0.0572 \\
\hline Death, n (\%) & 0 & $1(0.8)$ & $2(1.5)$ & 0.4275 \\
\hline \multicolumn{5}{|l|}{24 Months (Cumulative) } \\
\hline TVR, n (\%) & $15(12.6)$ & $18(15.1)$ & $47(34)$ & $<0.0001^{*}$ \\
\hline $\mathrm{MI}, \mathrm{n}$ & $3(2.5)$ & $6(5)$ & $13(9.4)$ & 0.0572 \\
\hline Death, $n$ & 0 & $1(0.8)$ & $2(1.5)$ & 0.4275 \\
\hline \multicolumn{5}{|l|}{84 Months (Cumulative) } \\
\hline TVR, n (\%) & $15(12.6)$ & $18(15.1)$ & $47(34)$ & $<0.0001^{*}$ \\
\hline $\mathrm{Ml}, \mathrm{n}$ & $3(2.5)$ & $6(5)$ & $13(9.4)$ & 0.0572 \\
\hline Death, n & 0 & $1(0.8)$ & $2(1.5)$ & 0.4275 \\
\hline Composite Ml or Death, n (\%) & $3(2.5)$ & $7(5.9)$ & $15(10.9)$ & $0.025 *$ \\
\hline
\end{tabular}

* $=$ Statistically significant

$\mathrm{MACE}=$ major adverse cardiac events

$\mathrm{MI}=$ myocardial infarction

TVR $=$ target vessel revascularization

\begin{tabular}{|c|c|c|c|c|}
\hline & & OR & $95 \% \mathrm{Cl}$ & P-VALUE \\
\hline \multirow{3}{*}{ MI } & B vs. A & 2.631 & $0.653-10.597$ & 0.174 \\
\hline & Cvs. A & 4.275 & $1.159-15.765$ & $0.029 *$ \\
\hline & B vs. C & 0.615 & $0.234-1.615$ & 0.324 \\
\hline \multirow{3}{*}{ TVR } & B vs. A & 1.985 & $0.954-4.43$ & 0.061 \\
\hline & Cvs. A & 3.321 & $1.671-6.597$ & $0.001^{*}$ \\
\hline & B vs. C & 0.623 & $0.343-1.130$ & 0.119 \\
\hline \multirow{3}{*}{ BLEEDING } & B vs. A & 3.429 & $1.398-8.41$ & $0.007^{*}$ \\
\hline & Cvs. A & 5.437 & $2.313-12.78$ & $<0.0001^{*}$ \\
\hline & B vs. C & 0.628 & $0.339-1.164$ & 0.139 \\
\hline
\end{tabular}

* $=$ Statistically significant

$\mathrm{MI}=$ myocardial infarction

$\mathrm{TVR}=$ target vessel revascularization

IV group C were 4 fold of that in-group A (OR 4.3, $95 \%$ CI: $1.16-15.77)(\mathrm{p}=0.029)$, and 1.60 times of those in group B (OR 1.6, 95\% CI: 0.23-1.62) (p = 0.324). Compared to group $A$, the odds of death or MI for group B were 2.6 times of that in-group A (OR 2.6, 95\% CI: 0.65- 10.60) ( $\mathrm{p}=0.174)$ (Figure 1).

Kaplan-Meier estimates for death or MI survival showed statistically significant difference among the groups $(\mathrm{P}=0.024)$ (Figure $2 \mathrm{~A})$. Compared with group $\mathrm{A}$, the cumulative odds of TVR were 3 -fold in group C (OR 3.3, 95\% CI 1.67-6.60, P = 0.001) and 2-fold in group-B (OR 1.99, 95\% CI 0.95- 4.43, $\mathrm{P}=0.061)$ (Figure 1). Kaplan-Meier estimates for TVR showed highly statistical difference in survival among the 3 groups $(\mathrm{P}<0.0001)$ (Figure 2B).

The risk of bleeding was significantly higher in group-C (OR 5.4, 95\% CI: 2.31-12.80) $(\mathrm{p}<0.0001)$ and group-B (OR 3.4, 95\% CI: 1.40-8.41) ( $\mathrm{p}=0.007)$ compared to group-A. All odds ratio were derived from the logistic regression equation. The frequency of rehospitalization was highly significantly lower in-group A (10.9\%) compared to group B (16.8\%) and group C (28\%) $\mathrm{P}=0.0009$ (Tables 3 and 4).

Subgroup analysis was done to look for any difference in composite of death or MI between the three groups in patients with diabetes, STEMI, NSTEMI/UA/ACS, and stable angina with positive thallium stress. There was no significant difference in the odds between the IV vs. the IC groups on the above-mentioned variables.

\section{Discussion}

Glycoprotein IIb/IIIa antagonists mitigate the thrombosis cascade and offer significant protection against ischemic complications of PCI with an absolute reduction of $1.5 \%$ to $6.5 \%$ in the 30 -day risk of death, MI, or urgent revascularization [28]. Treatment with 

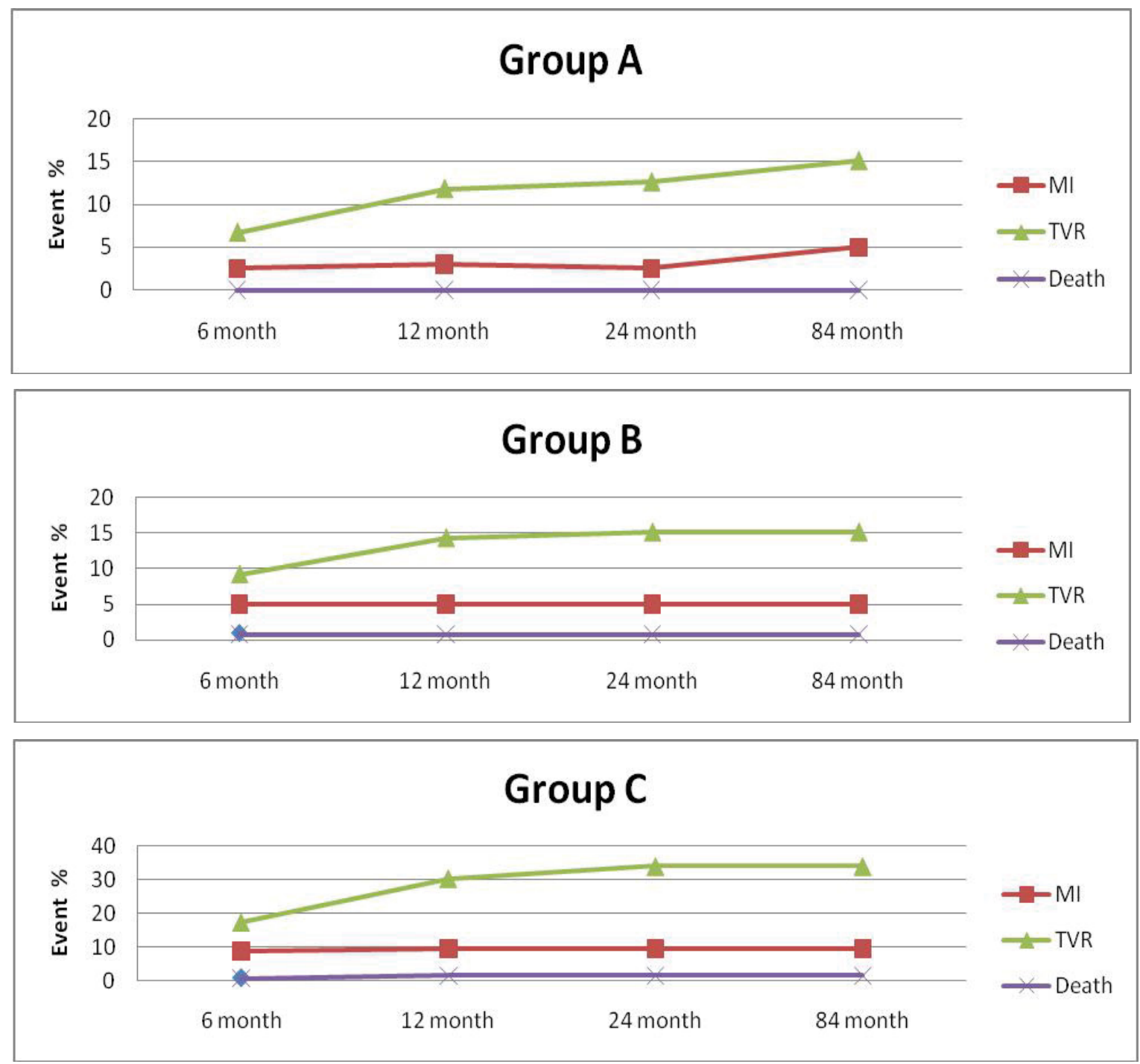

Figure 1: Group Event \% comparison over 84 months.

intravenous eptifibatide has been shown to reduce both short- and long-term adverse cardiac events when used during PCI [10,22-24,29]. In two small retrospective reports of IC bolus + IV administration for ST elevation MI [30] and non-ST elevation MI/unstable angina [31], eptifibatide showed few periprocedural adverse events. Yet, our study was first to examine the immediate, intermediate and long term results of safety and efficacy of eptifibatide IC only bolus administration during PCI.

Our study is an observational study compared clinical outcomes of three different modalities of eptifibatide administration used during PCI. This study included overall $52 \%$ diabetic patients with mainly type $\mathrm{B}$ and $\mathrm{C}$ lesions. Although, the IC group A included older patients with lowers left ventricular function, more coronary thrombi and lower TIMI III flow. Yet, the results suggest that the IC administration of bolus dose alone of eptifibatide may be safer and superior to the IV route demonstrating statistically significant difference in postprocedural bleeding, TVR and the composite endpoint of death or MI at 6,12, 24 and 84 months. The odds of having worse clinical outcomes were higher with IV bolus and/or continuous infusion groups (C, B). The high incidence of SAT and periprocedural MI in groups $B$ and $C$ probably can be explained in part by the high periprocedural bleeding incidence and episodes of decrease blood pressure and coronary perfusion. Potential advantages of IC route of administration include an increase in the local drug concentration by several order of magnitude thereby achieving higher levels of platelet GP IIb/IIIa receptor occupancy leading to thrombus, micro thrombi and vascular debris disaggregation. Other postulated benefits beside thrombus dissolution include improvement in distal microvascular circulation, endothelial function and 


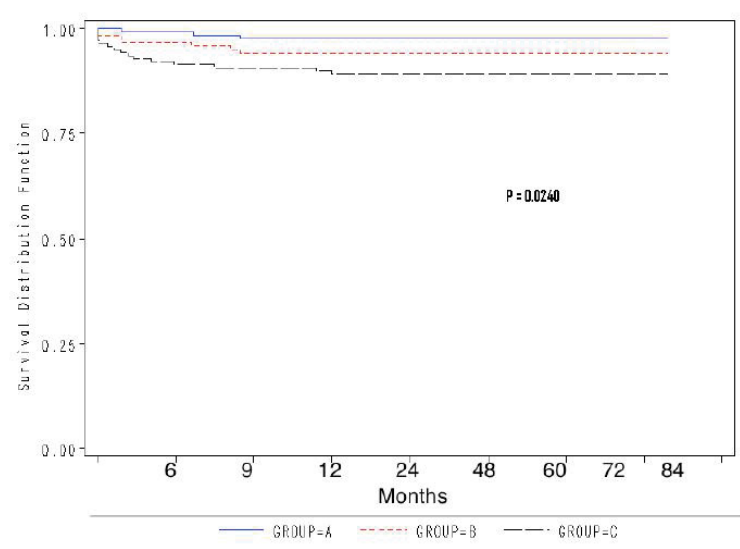

Figure 2A: Kaplan-Meier estimates for death or MI.

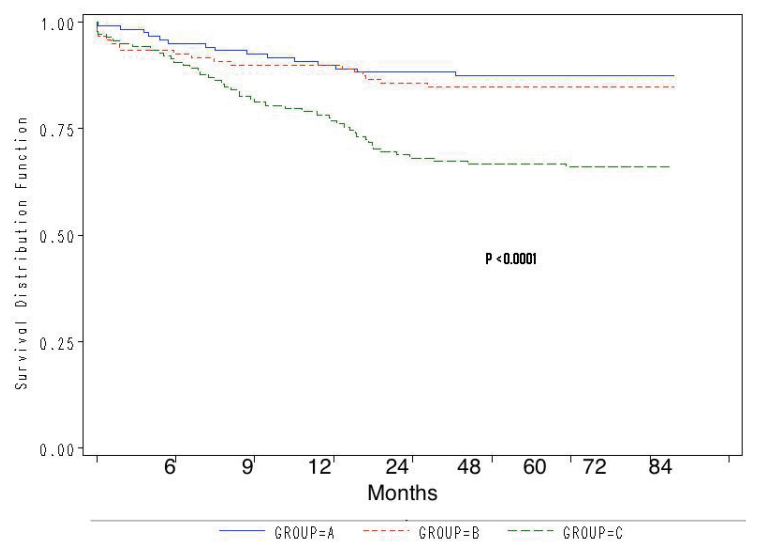

Figure 2B: Kaplan-Meier estimates for TVR. attenuation of post PCI inflammation. Eptifibatide is cleared predominately by the kidney, with no first pass hepatic metabolism, so IC administration eliminates the prolonged systemic concentration explaining the lower bleeding complications. Eptifibatide vehicle contains citric acid and sodium hydroxide to adjust the $\mathrm{PH}$ to 5.35 , which might cause a brief chest pain of variable intensity with transient $\mathrm{T}$ wave inversion during IC administration. The beneficial effects of IC bolus route of eptifibatide significantly reduced the periprocedural complications, TVR, rate of rehospitalization, and the risk of death or MI that extended up to 84 months, which may translate into cost saving.

Finally, with the recent introduction of the bioresorbable vascular scaffolds [32], we are planning to test if this IC drug delivery approach will hold similar or different outcomes.

The results of this study may have limitations, which include being an observational, retrospective registry analysis generating a hypothesis. The outcomes could have been affected by identified or hidden confounding factors similar to any non-randomized study.

\section{Conclusion}

The IC bolus alone application of eptifibatide during PCI may be safer and superior to the IV route, and continuous infusion may not be necessary. Large-scale prospective randomized trials including control groups are needed to further validate intracoronary application of this and other GP IIb/IIIa receptor antagonists.

\section{Executive summary}

Objectives: This study evaluated the immediate, intermediate and 7-year results of intracoronary (IC) eptifibatide administration during percutaneous coronary intervention $(\mathrm{PCl})$.

Background: Several studies tested intravenous (IV) bolus and continuous administration of eptifibatide during PCI. However, limited data is available considering giving eptifibatide as IC bolus alone during $\mathrm{PCl}$.

Methods: Clinical outcomes of 376 patients who received coronary stent(s) + eptifibatide by three applications during $\mathrm{PCl}$ and were followed up for over 84 months. Group A (119 patients) had IC eptifibatide bolus only; group B (119 patients) had IC bolus and IV infusion and group C (138 patients) had IV bolus + infusion. The standard two boluses of eptifibatide $180 \mathrm{mcg} / \mathrm{kg}$ were given either via IC or IV route and only groups B and C received IV infusion at $2 \mathrm{mcg} / \mathrm{kg} / \mathrm{min}$ for $18-24$ hours.

Results: There were 256 males and 120 females, mean age $57 \pm 11$. Among them $52 \%$ were diabetics. The 6, 12, 24, 84-month cumulative composite endpoint of death and myocardial infraction (MI) was lower in group A (2.5\%) compared to group C (10.8\%, OR 4.3, $\mathrm{p}=0.029)$, and group-B $(5.8 \%, \mathrm{OR} 2.6, \mathrm{p}=0.17)$. Compared to group-A, target vessel revascularization (TVR) was three fold in group-C (OR 3.3, $p=0.001)$ and two-fold in group-B $(O R$ 2.0, $p=$ 0.061). Bleeding was significantly higher in Group-C (OR 5.4, $p<0.0001)$ and Group-B (OR 3.4, $p=0.007)$ compared to Group-A. Re-hospitalization was significantly lower in group A (10.9\%) compared to group B (16.8\%) and group $C(28 \%)(P=0.0009)$.

Conclusion: The IC bolus alone application of eptifibatide may be safer and superior to the IV route, and continuous infusion may not be necessary. Large-scale prospective randomized trials are needed to further validate these findings. 


\section{References:}

1. Mizuno k, Satomura K, Miyamoto A, et al. Angioscopic evaluation of coronary-artery thrombi in acute coronary syndromes. N. Engl. J. Med.326, 287-291 (1992).

2. White CJ, Ramee SR, Collins TJ, et al. Coronary thrombi increase PTCA risk: angioscopy as a clinical tool. Circulation. 93, 253-258 (1996).

3. Fuster V, Badimon L, Badimon J, et al. The pathogenesis of coronary artery disease and the acute coronary syndromes. $N$. Engl. J. Med. 326, 242-250 (1992).

4. The EPIC Investigators. Use of a monoclonal antibody directed against the platelet glycoprotein IIb/IIIa receptor in high-risk coronary angioplasty: the EPIC Investigation. $N$. Engl. J. Med. 330, 956-961 (1994).

5. The EPILOG Investigators. Platelet glycoprotein IIb/IIIa receptor blockade and low-dose heparin during percutaneous coronary revascularization. N. Engl. J. Med. 336, 1689-1696 (1997).

6. The CAPTURE Investigators. Randomized placebo-controlled trial of abciximab before and during coronary intervention in refractory unstable angina: the CAPTURE study. Lancet. 349, 1429-1435 (1997).

7. The IMPACT-II Investigators. Randomized placebocontrolled trial of the effect of eptifibatide on complications of percutaneous coronary intervention: IMPACT-II. Integrilin to minimize Platelet Aggregation and Coronary Thrombosis-II. Lancet. 349, 1422-1428 (1997).

8. The RESTORE Investigators. Effects of platelet glycoprotein IIb/IIIa blockade with tirofiban on adverse cardiac events in patients with unstable angina or acute myocardial infarction undergoing coronary angioplasty: randomized efficacy study of tirofiban for outcomes and restenosis. Circulation. 96, 14451453 (1997).

9. The EPISTENT Investigators. Randomized placebo-controlled and balloon-angioplasty-controlled trial to assess safety of coronary stenting with use of platelet glycoprotein IIb/IIIa blockade. Lancet. 352, 87-92 (1998).

10. The ESPRIT Investigators. Novel dosing regimen of eptifibatide in planned coronary stent implantation (ESPRIT): a randomized, placebo-controlled trial. Lancet. 356, 2037 2044 (2000).

11. Kong DF, Califf RM, Miller DP, et al. Clinical outcomes of therapeutic agents that block the platelet glycoprotein IIb/IIIa integrin in ischemic heart disease. Circulation. 98, 2829-2835 (1998).

12. Anderson KM, Califf RM, Stone GW, et al. Long-term mortality benefit with abciximab in patients undergoing percutaneous coronary intervention. J. Am. Coll. Cardiol. 37, 2059-2065 (2001).

13. Karvouni E, Katritsis DG, Ioannidis JP. Intravenous glycoprotein IIb/IIIa receptor antagonists reduce mortality after percutaneous coronary interventions. J. Am. Coll. Cardiol. 41, 26-32 (2003)

14. Cohen DJ, Lincoff AM, Lavelle TA, et al. Economic evaluation of bivalirudin with provisional glycoprotein IIb/IIIa inhibition versus heparin with routine glycoprotein IIb/IIIa inhibition for percutaneous coronary intervention: results from the REPLACE-2 trial. J. Am. Coll. Cardiol. 44, 1792-1800 (2004).
15. Mitchel JF, Alberghini TV. Site-specific thrombolysis with Reopro. Circulation. 94, 1202 (1996).

16. Mitchel JF, Alberghini TV. Local delivery of repro: pharmacokinetics and effect on platelet deposition following balloon angioplasty. Circulation. 94, 1202 (1996).

17. Baily SR, O'Leary E, Chilton R. Angioscopic evaluation of site-specific administration of Reopro. Catheter. Cardiovasc. Diagn. 42, 181-184 (1997).

18. Bartorelli AL, Trabattoni D, Galli S, et al. Successful dissolution of occlusive coronary thrombus with local administration of abciximab during PTCA. Catheter. Cardiovasc. Interv. 48, 211-213 (1999).

19. Hassan W, Nambiar V, Al-Habeeb W, et al. Rescue in situ thrombolysis for acute coronary thromboembolism in an angiographically normal coronary artery. Saudi. Med. J. 25(12), 2007-2009 (2004).

20. Wohrle J, Grebe OC, Nusser T, et al. Reduction of major adverse cardiac events with intracoronary compared with intravenous bolus application of abciximab in patients with acute myocardial infarction or unstable angina undergoing coronary angioplasty. Circulation. 107, 1840-1843 (2003).

21. Kakkar AK, Moustapha A, Hanley HG, et al. Comparison of intracoronary vs. intravenous administration of abciximab in coronary stenting. Catheter. Cardiovasc. Interv. 61, 31-34 (2004).

22. O'Shea JC, Hafley G, Greenberg S, et al. Platelet glycoprotein IIb/IIIa integrin blockade with eptifibatide in coronary stent intervention. The ESPRIT trial: a randomized controlled trial. JAMA. 285, 2468-2473 (2001).

23. O'Shea JC, Buller C, Canton W, et al. Long term efficacy of platelet glycoprotein IIb/IIIa integrin blockade with eptifibatide in coronary stent intervention. JAMA. 287: 618621 (2001).

24. Hassan W, Al-Sergani H, Al Buraiki J, et al. Immediate and intermediate results of intracoronary stand-alone bolus administration of eptifibatide during coronary intervention (ICE) study. Am. Heart. J. 154, 345-351 (2007).

25. Hassan WM, Flaker GC, Feutz C, et al. Improved anticoagulation with a weight-adjusted heparin nomogram in patients with acute coronary syndromes: A randomized trial. $J$. Thrombosis. Thrombolysis. 2, 245-249 (1995).

26. Rao AK, Pratt C, Berke A, et al. Thrombolysis in Myocardial Infarction (TIMI) trial: phase 1, hemorrhagic manifestations and changes in plasma fibrinogen and the fibrinolytic system in patients treated with recombinant tissue plasminogen activator and streptokinase. J. Am. Coll. Cardiol. 11, 1-11 (1988).

27. Lincoff AM, Califf RM, Moliterno DJ, et al. Complementary clinical benefits of coronary-artery stenting and blockade of platelet glycoprotein IIb/IIIa receptors: evaluation of platelet IIb/IIIa inhibition in stenting investigators. N. Engl. J. Med. 341: 319-327 (1999).

28. Lincoff AM, Califf RM, Topol EJ. Platelet glycoprotein IIb/ IIIa receptor blockade in coronary artery disease. J. Am. Coll. Cardiol. 35, 1103-1115 (2000).

29. Tcheng JE, Strony J, Lorenz TJ, O'Shea JC. ESPRIT in context: pharmacology matters! Eur. Heart. J. 22, 1965-1967 (2001). 
30. Pinto DS, Kirtane AJ, Ruocco NA, et al. Administration of intracoronary eptifibatide during ST-elevation myocardial infarction. Am. J. Cardiol. 95(11), 1494-1497 (2005).

31. Deibele AJ, Kirtane AJ, Pinto DS, et al. Intracoronary bolus administration of eptifibatide during percutaneous coronary stenting for non ST elevation myocardial infarction and unstable angina. J. Thromb. Thrombolysis. 22, 47-50 (2006).

32. Devito F, Zito A, Dachille A, et al. Bioresorbable vascular scaffolds: design, clinical trials, and current applications. Coron. Artery. Dis. 27:151-158 (2016). 